\title{
Birkhoff's aesthetics, Arnheim's entropy. Some remarks on complexity and fuzzy entropy in arts
}

\author{
Marco Elio Tabacchi ${ }^{1}{ }^{2}$ Settimo Termini ${ }^{1},{ }^{3}$ \\ ${ }^{1}$ DMI, Università degli Studi di Palermo \\ E-mail: marcoelio.tabacchi@unipa.it \\ ${ }^{2}$ Istituto Nazionale di Ricerche Demopolis, Italy \\ ${ }^{3}$ ECSC, Mieres (Spain) E-mail: settimo.termini@unipa.it
}

Received 9 July 2015

Accepted 5 October 2015

\begin{abstract}
A judgement of aesthetic in arts is, by sheer consensus, a daunting task that requires evaluation of a whole host of endogenous and exogenous cultural factors. A few of them can actually provide very useful hints in tackling foundational problems in Information Science in a more natural setting than what is usually provided by a typical engineering stance. This interaction can however work the other way about, as instruments from the Information and Computer Science toolkit may help in focusing the less explored features of art and its evaluation. When all the social, historical, hermeneutical and political considerations are stripped from the living flesh of the piece, we lose most of what differentiates creation from description. This notwithstanding, or maybe exactly for this reason, measuring structures is still an important element of artistic judgement, and the folk concept that beauty stems from some sort of order/chaos relationship, formalized by G. D. Birkhoff as the aesthetic measure, requires an adequate and consistent quantification of both factors. Old and new approaches to the problem generally resort to classical definitions of information and entropy (Shannon entropy, Kolmogorov-Solomonoff complexity) and their derivatives, neglecting the fact that compactness and repetition have a different value in arts than in information theory, a "confusion of our languages" already noted by R. Arnheim. In this paper we discuss a tiny fragment of the general and wide mesh of interactions between information sciences and humanities: a possible, fruitful interaction among such different topics as fuzziness and art, dealing with similarities and differences in measuring fuzziness and information, and the relationship between the informal notion of information and the measures of fuzziness.
\end{abstract}

Keywords: Fuzziness, Art, Complexity evaluation, Fuzzy Entropy

\section{Introduction}

Art, aesthetic theories seem to be a very good place to test the possible, fruitful interaction between the new (and technically and conceptually innovative) tools provided in the last decades by Computer and Information sciences on one side and a few deep questions asked by Humanities, which apparently seem to be quite impervious to the enquiries carried on with the means of traditional scientific methodology. The very limited aim of the present paper is to test this kind of interaction between fuzziness (measures of fuzziness and information) and art (aesthetic theories), using as a starting point the reconciliation of two old and seemingly contrasting approaches to the problem of aesthetic evaluation. We have tried 
to condense in the title of this paper some remarks that should be critically reviewed: there are similarities and differences when trying to measure fuzziness and information, and there is some sort of relationship between the informal notion of information and the measures of fuzziness.

The present paper is epistemologically based on some recent reflections of the authors ${ }^{46}$, stimulated by the attempt at answering to such challenging questions, also of epistemological type, as the ones Lotfi Zadeh posed by shifting from the basic idea of fuzzy sets as an extended characteristic function, to linguistic variables and subsequently to other levels until arriving at the more recent ideas of computing with words and taking perceptions as a starting point for scientific investigations. This is a slippery road indeed: we must take into account all the new questions and problems that have arisen in taking seriously some innovative questions as the ones mentioned above, but also travel into the no man's land between the use of such notions as information and complexity in fields outside the original domain in which such notions originated. However, we firmly believe that the fact of confronting these questions in a common setting with all the controversial points, weakness, conceptual and technical difficulties present together on the stage can help with focusing the crucial points and to deal with them in a more effective way.

Also the land of the application of mathematical tools and techniques to aesthetic questions is very slippery. We do not refer to such classical questions as the ones brilliantly described by Hermann Weyl in his classical book Symmetry ${ }^{47}$, but to the attempts at capturing some hard to focus questions, such as for instance the one of formally describing Information in Art, or the one of providing specific, quantitative aesthetic measures. This choice of examples is not casual: we explicitly want to start from them for our general reflection, evolving for what at this stage may seems just a slogan to promote integration between fuzziness and art.

A number of endogenous and exogenous factors are implied in any meaningful evaluation of beauty in art - among them cultural differences, perceptive skills, common knowledge - but the repeating theme of aesthetic judgement through analysis of visual factors often pops out in some algorithmic fashion. To address the role of fuzziness in such context, we shall start from two (apparently) opposed positions on the possibility of formally approaching theoretical questions regarding art: the ones heralded by George David Birkhoff ${ }^{5}$ and Rudolf Arnheim 1 . Let us commence by asking in a very direct and sketchy way if it is considered possible, and frankly acceptable, to devise and use quantitative measures for such intriguing problems as the ones posed by Art. The relationships between Art and Science or better, between Art and the use of scientific methodology for investigating its features have often be hostile. John Barrow has mused on whether the relationships between Art and Science could be defined as Liaisons Dangereuses ${ }^{2}$ In fact, he affirms that:

Most artists are very nervous of scientific analysis. They feel it destroys something about the human aspect of creativity. The fear (possibly real) of unsubtle reductionism - music is nothing but the trace of an air pressure curve - is widespread. As a corollary, one finds the equally pernicious view that science has nothing to offer the arts, that they must transcend all attempts to capture them.

This is indeed the present situation, and we think that we could pay attention to conceptual problems as well as take into consideration especially those clear-cut proposals that allows to be looked at clearly. One could perhaps also wonder why in a preliminary investigation, whose aim is only to charter the territory, such a preeminent space has been given to two specific scholars, Arnheim and Birkhoff. And all of this even before confronting the main question, namely, the role that fuzzy approach, among others, can play in trying to better capture, by using a scientific methodology (better, the methodology of hard sciences), some meaningful aspects of aesthetic experience. Here is the right moment to stress again how in our point of view the true interaction between Humans and Computer/Information Sciences produces really innovative and crucial questions that both sides should trea- 
sure. The specific points hereby discussed are, then, even more meaningful that their face value, as they hint at much more general questions and open problems. The importance and greatness of these two scholars is out of question, but we have explicitly referred to them not only for their important contributions, but mainly for what they can represent in this kind of studies. So we shall use them as symbols of the two crucial points, we have referred to previously. Arnheim will be taken as a sort of banner of the request that, when trying to applying (important) results obtained in a specific field of investigation outside the domain in which they have been conceived and developed, we have the duty to carefully control the way in which these results and techniques are used and managed in the new domain. This great care must also be extended from the control of the correct application of the technical aspects in the new field to a careful analysis of conceptual questions, in order to scrutinize whether the use of the informal notion to which the theory is applied corresponds correctly to the intended meaning of the same notion in the field in which it was informally used before. This is crucial, in our view, for a proper testing of the correctness of any attempt to apply tools and theories from the scientific world to questions from the human domain. We have postulated elsewhere $\left({ }^{46}\right.$, but see also ${ }^{42,44,45}$ ) that an important help in this direction can be provided by Carnap's analysis of the procedure of explication ${ }^{14}$. Regarding Birkhoff, we shall consider him as a sort of banner of another attitude, which in our view is also of crucial importance. The courage of advancing very precise, specific and clear-cut proposals. This is not only a sign of intellectual integrity, which deserves the utmost consideration, but is also crucial for any meaningful advancement: despite critics abounded since about its inception, many of them reasonable (but it is much easier to criticize clear-cut proposals than vague hypothesis), Birkhoff's idea was still used as a basis for aesthetic measures' models forty years after its publishing, a testimony to its boldness.

The rest of this paper is organized as follows: in section 2 we will briefly describe Birkhoff's idea about a formal, mathematical way to approach a few aspects of aesthetic experience. In section 3, we shall present some elements from the critical analysis provided by Rudolf Arnheim which are relevant for the problems discussed here. Section 4 will discuss about how Birkhoff's idea (and Arnheim's criticism) have evolved, aided also by new technological means, by focusing on a few papers which seems paradigmatic of the epistemological questions we want to discuss. After a brief introduction to measures of fuzziness, in section 5 we will discuss and comment on the conceptual innovations provided by replacing classical Shannonian information measures with a more flexible tool based on a fuzzy approach. In our view, this preliminary results validate the strategy of looking for a new approach and technique every time a methodological questions arises from a direct application of a formal tool to a problem, and this same problem is ill defined from the point of view of the implicit philosophy of the formal technique proposed for its solution. In order to open a wide discussion, we have deliberately chosen a colloquial style, and reduced to a minimum the technical details.

\section{George David Birkhoff and the necessity of measuring}

The concept of unity in variety, a cornerstone of human judgment of beauty, is as old as the human interest in beauty and aesthetic values. According to Bosanquet ${ }^{6}$ :

[t]he relation of whole to part - a slightly more concrete expression for unity in variety - has never been more perfectly elucidated and more justly appreciated than by Plato and Aristotele, and it is in recognizing the satisfaction afforded to the mind by the sensuous or imaginative embodiment of this relation that they make a first step in genuine æsthetic analysis.

Most aesthetic theories in cognitive science, from the seminal research of Fechner ${ }^{21}$, and up to the most comprehensive (and modern, in a sense) model by Berlyne ${ }^{4}$, consider the idea of beauty stemming out from some sort of relationship between 
order and complexity as axiomatic. But the analysis in these theories is eminently qualitative, more directed toward a comparative subjective judgment - and the following psychological evaluation of the observer both in a personal and a cultural domain, than an absolute evaluation of aesthetic factors considered as intrinsic factors of the structure being examined. George Birkhoff, the renowned mathematician of ergodic theory fame with a well known and documented interest in artistic disciplines offers a breakthrough proposition in his pioneering 1932 book Aesthetic Measures ${ }^{5}$ : for each subdomain of the artistic endeavor, the aesthetic value of an opus, or aesthetic measure, is a direct function of the ratio between its order and its complexity. This synthetic and yet comprehensive definition moves the gist of aesthetic evaluation from the original qualitative and subjective stance to the more rigorous problem of finding

[w]ithin each class of aesthetic objects, [...] the order $O$ and the complexity $C$ so that their ratio $M=O / C$ yields the aesthetic measure of any object in the class.

At large, complexity is a function of the number of objects or elements composing the whole, while order is a function of the number of regularities found in the objects or in their arrangement. But this is a very general attribution, more declarative than descriptive; each and any discipline needs to define its own terms for Order and Complexity. As such the expansion of these general terms is very discipline dependent: in a very simple environment introduced by Birkhoff, evaluation of the aesthetics of simple polygons, these concepts are evaluated including factors as vertical and radial symmetry (see 52 for the idea of symmetry as a visual feature, and idea dear to Birkhoff, and ${ }^{30}$ for the contribution of symmetry to shape formation), the number of sides (for Complexity), and stability against an imaginary plane (for Order). While in the polygons example this choice of parameters brings out the predictable election of the square as the most aesthetically pleasing - something which will not even be supported by human validation in further works (the most comprehensive of which is ${ }^{20}$ ), in another example re- garding the silhouettes of Chinese vases the outcome is different. With Scha and Bod ${ }^{33}$,

[t]he formula behaves in a more interesting way now. The Esthetic Measure now correlates with a quality of "elegance", rather than a trivial property of orderedness.

In order to demonstrate how the simple principle can be applied not only to the visual domain, but also to less obvious artistic disciplines, Birkhoff goes on by analyzing music and poetry. While music, considered in its mono-dimensionality can at first seem a much easier target (and sometimes is - see ${ }^{8,13}$ for a comparison between music and paintings complexity evaluation which could be used in an aesthetic setting), Birkhoff derivation of a valid formula for harmony and melody passes through a series of complicated descriptions, which the author will keep in mind when dealing with poetry. The aesthetic measure was strongly criticized since about its inception. Already in 1939 D. J. Wilson ${ }^{48}$ affirmed that the hypothesis of Birkhoff that his a priori measure is a true measure of aesthetic values as far as polygons and geometrical figures are concerned was not supported by the results of his investigation, adding that "it is suggested, furthermore, that if the hypothesis fails here, it will fail a fortiori in more complex realms of art". Birkhoff's former PhD student C.A. Garabedian, in reviewing the book ${ }^{23}$, was even more caustic:

the book, closes with a description far from convincing to us - of the services which the author believes his theory capable of performing both in the aesthetic experience and in the creative process. [...] We affirm that the intuitive and the conceptual are indisputably distinct [and that] until we totally separate music and mathematics, art and science, and proceed on the basis of their parity, we shall not be making headway.

This tendency continued up to the present times, and some of the critiques leveled against the existence of a purely formal way of judging aesthetics have 
their value (in the next section we will discuss Arnheim's stance). Still, as stated in the introduction, forty years after the book was published, theories were still based on Birkhoff's intuition, and as of today, most of the papers dealing with the topic start discussing it, and rarely for historical reasons.

\section{Rudolf Arnheim: entropy, orderliness, order and tension}

Forty years after Birkhoff, Rudolf Arnheim returns to the same topic by discussing the role of order (and disorder) in esthetics, in his aptly titled "Entropy and Art: an essay on disorder and order" ${ }^{1}$, a svelte booklet that should be required reading for anyone in humanities willing to approach the topic of entropy without prerequisites and preconceptions (and no formulas either). In the first of a two part program, Arnheim tackles the problem of defining order in contexts as diverse as art, philosophy, psychology, evolution, physics - a field from which he takes the original idea of entropy in order to extend it to the human side of the equation. In this truly interdisciplinary tentative of injecting the rigor and precision of hard sciences into the domain of humanities, is an (apparently) passing remark that seems mostly connected with the problem of aesthetic computation based on a order/complexity ratio:

the entropy principle defines order simply as an improbable arrangement of elements, regardless of whether the macro-shape of this arrangement is beautifully structured or most arbitrarily deformed; and it calls disorder the dissolution of such an improbable arrangement.

In Arnheim's view this is clearly unacceptable:

The absurd consequences of neglecting structure but using the concept of order just the same are evident if one examines the present terminology of information theory. Here order is described as the carrier of information, because information is defined as the opposite of entropy, and entropy is a measure of disorder. To transmit information means to induce order. [But] since entropy grows with the probability of a state of affairs, information does the opposite: it increases with its improbability. [...] The conclusion is that total disorder provides a maximum of information; and since information is measured by order, a maximum of order is conveyed by a maximum of disorder. Obviously, this is a Babylonian muddle. Somebody or something has confounded our language.

The concepts of order and entropy have no sense if they are not viewed in a framework where aesthetic values are not only generated by the components of a determined stimulus, but are also implicit in its global structure. Numbers relative to order and complexity alone cannot describe aesthetics we need a better definition of what order means, a clear description of how order contributes to beauty, and a more comprehensive view of what all the components of the equation should contribute to fit together. Therefore, in the second part the aim of the essay seems to remove order as the most sought instance in human aesthetic cognitive effort, in order to subjugate it to a more detailed combination of stimuli such as entropy, tension reduction and homeostasis, collectively called anabolic creation. Here, in citing Lovejoy ${ }^{26}$ Arnheim deals with the apparent contrast between complexity and simplicity, and catches a new reference to an old friend - unity in variety:

And it is precisely because "God makes the greatest number of things that he can," that the laws of nature have to be as simple as possible. [...] The arts, as a reflection of human existence at its highest, have always and spontaneously lived up to this demand of plenitude. [...] There is no need to rediscover here the ancient formula of "unity in variety" [as] the pleasures of the least effort are to be derived not from the simplest available patterns but presuppose the "fullest actions" of the perti- 
nent mental faculties, the "maximum of stimulation," drawing from the nervous system "the maximum amount of energy". [...] Even the most traditional aesthetic taste does not limit itself, in the more sensitive observers, to considering beauty exclusively as the absence of distorting stresses.

Thus the reference to the problem of aesthetic evaluation becomes more explicit, even at the point of directly citing Birkhoff ${ }^{5}$ :

[h]omogeneity is the simplest possible level of order because it is the most elementary structural scheme that can be subjected to ordering. Orderliness comes in degrees; order comes in levels. A structure can be more or less orderly at any level of complexity. The level of ordered complexity is the level of order. The "aesthetic measure" at which George Birkhoff aimed was merely a measure of order, derived from the relation between orderliness and complexity. Order, I shall suggest, is a necessary although not a sufficient condition of aesthetic excellence.

Such statement, which may at first glance seem as a harsh criticism of the simplification operated by Birkhoff $^{5}$, is instead a proposal to overcome the already mentioned limit of his theory: a preference for simplicity which leads to compute beauty in banality (we will see in the next section that this problem will carry on to the contemporary computational aesthetic models). With the inward directed order (and orderliness, its instantiation) has to come an outward force, that Arnheim calls disorderly destruction, the combination of which promote the increase of entropy, and with its equilibrium, beauty. Arnheim notes on Winkelmann appreciation of a Hellenistic statue, "the visual tension animating the human form was to him as indispensable for beauty as were simplicity and quietness". Arnheim has found the right cure for one of Computational aesthetic's main ailments: complexity is not just a counterpart to order, a subtractive element toward the path of beauty, but part of a whole, complex dynamic made of tension and order, chaos and simplicity. The reason why this still is not incorporated in the models (and, probably, why Arnheim never made a more clear-cut, measurable proposition) is to be searched in the tools used to derive the models. Classical logic, sharp measures are not flexible enough to capture the sensibility of such an interplay. In Section 5 we will discuss methodologies which seem to us more appropriate to this interdisciplinary task.

\section{Computational Aesthetic}

\subsection{Birkhoff's legacy}

Thus, Birkhoff sharp formalization of the unity in variety principle stands as the cornerstone of what we call Computational Aesthetics, or the science of deriving a beauty judgment from intrinsic, measurable factors. While Computational Aesthetics is today a widely accepted label, this discipline has a long standing tradition of labeling itself differently according to the prevalent bent of the times, a short chronicle of which is duly recorded in ${ }^{25}$. The term is due to Scha and Bod - the first appearance in print is from the already cited ${ }^{33}$ in its English translation. This discipline is still producing interesting results as of today, and while its different incarnations, from Bense's Information Aesthetics to Stiny and Gips' Algorithmic Aesthetics ${ }^{37}$, have gone a long way in refining the computational aspect and in selecting the modeling parameters, they all share the original intuition expressed by $M=O / C$, and the target, expressed by Birkhoff in his concluding remarks:

it seems almost obvious that aesthetics, if it is to be scientific, must be approached from the analytic point of view and must concern itself chiefly with the formal aspects of art.

\subsection{Bense and Information Aesthetics}

While this is not the place for an excursus in computational aesthetics (for a short but very well written one see the already mentioned ${ }^{33}$ ), me must briefly discuss Information Aesthetic. 
Birkhoff's book is published in 1932. Gödel's incompleteness theorems have been published just the year before, Turing still a student at King's College, Shannon an adolescent, the term Cybernetics will be coined sixteen years after, AI is twenty four years away. In this context, by necessity, Birkhoff's models had to be purely mathematic (even worse, when dealing with bidimensional data, such as images made of pixels, or geometric). This is an obvious limitation: defining complexity starting by simple geometric entities or by durations and pauses, as in music and poetry, is clearly not easy nor rewarding.

The sixties were the right times to incorporate into aesthetic evaluation some knowledge from the booming world of computer science, and the fruit of this is Bense's Information Aesthetic ${ }^{3}$. In Bense's model, Order and Complexity are no more just abstract concepts, but they are linked to the perceived content of information in the stimulus, computed following Shannon Information Theory. To be a little more precise, the image is considered as formed by patterns made of a finite number of symbols arranged in a hierarchical way depending on size (Bense considers this a general method, preconizing in some way the digital image and the pyramidal approach of image analysis) and these patterns form in turn super-symbols by aggregation. At each level the quantity and diversity of symbols is accounted for, and the more these super-symbols repeat themselves, the less the information content of the image is, as the image itself can be described more compactly in term of the super-symbols. Aesthetic measure following this model is the ratio between the simplification obtained by describing the image in term of super-symbols and the original information content of the image when described in term of symbols. As the model is general, it can be applied to any kind of signal that can be decomposed in symbols, and re-composed in super-symbols; this also includes music, poetry and texts.

One glaring defect of the Information approach is that while correctly assigning lower scores to random stimuli, as they are devoid of useful information, it has a preference - shared with Birkhoff original model - for the simplest stimuli, such as images composed of repeating patterns. Such images are clearly boring, and much less interesting in term of aesthetic than many less-regular ones (we duly note that the arguments used in this criticism will resound much later in Wolfram's classification ${ }^{49}$ ). Aside from that, the real merit in Bense's approach is to have expanded Birkhoff's original idea toward Information theory, and as such have paved the way for computer scientists to embrace computational aesthetics (and for humanists to learn some basic concepts from hard science).

\subsection{A contemporary measures of aesthetic}

Being based on patterns, Bense's approach lends naturally to the transition from analogic to digital images, from colours to pixels, from unity to segmentation. Among the papers taking this path, one by Rigau et al. $\left({ }^{31}\right.$, expanded in $\left.{ }^{32}\right)$ is interesting for its use of a concept called physical entropy. Zurek defines physical entropy as the sum of the missing information and the algorithmic information content of available data ${ }^{53}$, as such putting together Shannon Information and Kolmogorov-Solomonoff Complexity. The concept of physical entropy is useful in describing dynamic systems, where measurements of the system properties are possible, and each measurement can increase the knowledge about the system itself. Using this concept, Rigau et al. propose a new version of Bense's measure, given by the ratio between the reduction of uncertainty (or a measure of the compression achieved) and the initial information content of the image. This measure is applied to different paintings, obtaining result in line with the work of Birkhoff and Bense: Mondrian, with his flat (and highly compressible) surfaces gets the highest score, while Pollock and his (seemingly) randomly painted surfaces gets the lowest.

While not unique nor necessarily indicative, the previous example represents in our view the current trends in computational aesthetics: when considering the evaluation of complexity, a classical concept of information is recalled, and classical measures are employed. This is one more reason to advocate taking a new path for tackling an old problem, and to suggest that there is space for a fruitful integration of fuzziness and art, something we will discuss in 
the next section.

\section{Fuzziness, or how to measure in the real world}

In this Section, providing a few reference for classical results, we shall try to present them in a way that clarifies our thesis that, when seen in its innovative aspects, fuzziness can provide a functional alternative to measuring sharp information in order to determine a complexity factor for aesthetic measurement.

\subsection{Modelling fuzziness}

Let us now turn to fuzzy sets. Although what we shall now recall can be well known to many readers, we think that it can be useful to provide also a few fragments of the early history in order to assess the conceptual framework inside which we propose to move our discussion. The concept of "fuzzy set" was introduced by Lotfi Zadeh in $1965^{51}$, but he had already outlined the general idea in some previous papers also under some different names ${ }^{50}$. For a survey of this early history one can read the paper by Brian Gaines ${ }^{22}$ which highlights also many other interesting aspects and questions. However, the basic idea, in different settings and with different terminologies, had been anticipated by other scholars. For instance - although constrained by a probabilistic, however non dogmatic, vision - it can be traced in some brief notes by Karl Menger ${ }^{27,28}$ as well as in some remarks by Thoralf Skolem ${ }^{36}$. Also the consistency profiles by Max Black are a sort of anticipation of the idea of fuzzy set ${ }^{35}$.

Let us observe that all these proposals remained confined in small niches, without any impact outside very restricted audiences. A much more detailed description of this events has been provided by Rudolf Seising ${ }^{34}$. So an important question emerges and it is related to understand the reasons why among all these attempts (and many others) the one of Zadeh is the unique which had a sudden impact, coming to involve in a short period of time greater and greater numbers of researchers, so the unique successful from a sociological point of view. Joseph Goguen has arrived to connect the general osophy underlying Zadeh's proposal to "the social nature of truth" ${ }^{24}$. Another interesting phenomenon to be analyzed by historians of ideas is the apparently easier acceptance in eastern countries (China, Japan and India) of his idea. One main reason may be that among all these attempts, Zadeh's proposal is the only one bravely departing from the classical tradition of the use of mathematics without bothering too much about possible criticism and consequences of the perilous footpaths he was suggesting; the other proposals - however innovative - moved along recognized boundaries. Zadeh's is also the only approach motivated, from the origin, by applications. These aspects are of interest here for the relationship with aesthetic experience, since we believe that there is a lot in common between technology and humanities: more, in some cases, than between technology and hard sciences ${ }^{40}$.

These apparently sparse considerations, point to a crucial, foundational point: the specificity of the interactions among technology, humanities and (theoretical) hard sciences. This seem not only to present new and important problems to solve, but also to show mutual connections which lead into unforeseeable directions. Also in this sense, fuzziness is really a new notion that can play a crucial role in assessing these new questions.

\subsection{Measuring fuzziness}

In the following we shall present a brief overview of the essential points of the theory of Measures of Fuzziness $^{41}$, which is one of the various different facets that the notion of fuzziness presents. In this brief summary we shall not provide details regarding proofs and purely technical developments for which the reader is referred to the literature. We shall however introduce, with a minimum of detail, a bit of formalism (in particular, some of the axioms of the measures of fuzziness and a few examples), differently from what we have done in the previous Sections. Our aim, in fact, is to show to the reader why we think that a different path towards the formalization of the notion of entropy and information can reduce, at least, the perplexities of Arnheim who has so lucidly criticized an uncritical and mechanical application of Shannon's theory to aestetic questions. 
Along the presentation some comments will point out where the interaction between informal ideas and technicalities seems to be stronger. Let us finally observe that we shall not significantly refer to the literature connected to the theory of measures of fuzziness subsequent the eighties of last Century, since Ebank's paper ${ }^{19}$ provides a complete classification of all the possible measures of fuzziness in the standard interpretation. Ebanks' work, in a sense, put the final words on the standard theory. Possible paths that could be followed in order to extend the theory are indicated in ${ }^{18}$, but these developments do not seem (at least to the authors) particularly meaningful for the topics discussed in this paper.

Let's recall some elementary definitions. Given a universe $X$, a fuzzy set is any mapping $f: X \rightarrow[0,1]$, that is, a rule that assign to each element of $X$ a value in $[0,1]$, its "degree of membership". Let us denote by $\mathscr{L}(X)$ the class of all fuzzy sets on $X$, i. e. all the applications from $X$ to $[0,1]$.

Given now $\mathscr{L}(X)$, i. e. the class of all fuzzy sets, we want to introduce a way of measuring "how fuzzy" a fuzzy set is, namely how much it departs from a classical characteristic function which exactly inform us about the sharp belonging (or not belonging) of an element of $X$ to a given subset of the same $X$.

Just for outlining how this can be done we need to introduce a few additional technical definitions.

It is possible to introduce a structure of a (distributive) lattice in $\mathscr{L}(X)$ by means of the binary operations $\vee$ and $\wedge$ which associate to every pair of elements $f$ and $g$ of $\mathscr{L}(X)$ the elements $f \vee g$ and $f \wedge g$ of $\mathscr{L}(X)$ defined as follows: for any $x \in X$

$$
\begin{aligned}
& (f \vee g)(x)=\max \{f(x), g(x)\} \\
& (f \wedge g)(x)=\min \{f(x), g(x)\}
\end{aligned}
$$

Furthermore, for any $f$ in $X$, one can introduce the so called negation $f^{\prime}$ defined as: for any $x$ in $X$

$$
f^{\prime}(x)=1-f(x)
$$

Can we judge whether a fuzzy set is sharper of another fuzzy set? A way for doing this is by using the following (partial) order relation.
Let us now introduce in the interval $I=[0,1]$ the partial order relation $\leqslant$ defined, for any $x, y \in I$, as

$$
x \leqslant y \Leftrightarrow x \leqslant y \leqslant \frac{1}{2} \vee x \geqslant y \geqslant \frac{1}{2}
$$

This relation can be extended, point by point, to $\mathscr{L}(X)$ as follows: for any $f$ and $g$ in $X$,

$$
f \leqslant^{\prime} g \Leftrightarrow \forall x \in X\left[f(x) \leqslant \leqslant^{\prime} g(x)\right]
$$

If $f \leqslant^{\prime} g$ and $f \neq g$ we shall say that $f$ is sharper than $g$.

A measure of fuzziness or entropy measure $h$ is simply a functional $h: X \rightarrow R+$, where $R+$ denotes non-negative reals, satisfying some conditions (see $16,15,17$ for more details). A feature of the theory is that the conditions to be imposed can depend on the system under consideration. So we must not require the same conditions once and for all, but list some reasonable requirements and check - in the considered system - which, in the list, must be required and imposed. However we can reasonable assume that there are some basic assumptions which should always hold. In particular:

- the concept of fuzziness disappears in the classical case,

- the fuzziness be maximum when it is impossible to distinguish between a fuzzy set and its negation

- this measure must certify quantitatively the sharpness of a fuzzy set, described starting from the order relation $\leqslant$.

Such conditions have the formal counterparts in the basic axioms which any measures of fuzziness must necessarily satisfy, which are as follows:

- (a) $h(f)=0$ if and only if $f$ is a classic characteristic function.

- (b) $h(f)$ attains its maximum value if and only if $f=f^{\prime}$.

- (c) $h$ is isotone with respect to the order $\leqslant^{\prime}$, that is, if $f \leqslant^{\prime} g$ then $h(f) \leqslant h(g)$

The complete list of axioms can be found in 19 and ${ }^{18}$. Let us observe that Ebanks has introduced a technical axiom (not showing interesting conceptual 
motivation) in order to arrive at a unique measure of fuzziness.

Examples of simple measures of fuzziness (besides the well known measure of Shannon) are the following ones:

$$
\begin{gathered}
\sigma(f)=\sum_{x \in X} f(x)(1-f(x)) \\
u(f)=\sum_{x \in X} \min \{f(x), 1-f(x)\}
\end{gathered}
$$

Measure $\sigma(f)$ is formally identical to the sum of the variance of the random variables $\xi(x)$ assuming the values 1 and 0 with probability $f(x)$ and $1-f(x)$, respectively. Measure $\sigma(f)$ has been frequently used in statistical pattern recognition for representing the information content of a given image, as it doesn't lend to the same overrepresentation of marginal data patterns as Shannon's measure.

The measures of fuzziness $\sigma(f)$ and $u(f)$ as well as the logarithmic one satisfy almost all the axioms listed in the references given, while an example of a measure which does not satisfy one of the additional axiom (the valuation property) is given by $[\sigma(f)]^{2}$.

All these examples, however simple, are useful for investigating in vivo the interactions between "(informal) concepts" and "(possible) formalizations". If one imposes all the six proposed axioms the unique measure of fuzziness of a fuzzy set $f$ is provided by $\sigma(f)$ as introduced above.

\subsection{A reason for fuzzy measures}

Measures such as $\sigma(f), u(f)$ (introduced above) and the many variations based on the same idea thereof are not just a theoretical cunning device: their implementation is numerically feasible and can be adapted to a different family of problems in an ample variety of settings. Just in order to give a concrete idea of their implementation we will briefly recall previous work done on two experiments devoted to the evaluation of visual complexity ${ }^{11,13}$ to which we point the reader for full details. In ${ }^{10}$ a variety of painting with different kind of content, such as human figures, landscapes and natural settings, and schematic representations are graded for their perceived complexity by a host of human subjects through indirect estimation. A digital evaluation is then carried out by searching in the images for local symmetries using the Digital Symmetry Transform, and then applying some direct variations of $\sigma(f)$, e.g.

$$
\sigma^{\prime}(f)=\sum_{x \in X} 4 f(x)(1-f(x))
$$

The results obtained by this process are very similar to those from human performances, in contrast with the application of Shannon's entropy which, being targeted to purely statistical aspects of the images cannot capture the complexity borne out from the significative elements of it. A further variant of $\sigma(f)$ has been usefully employed in another evaluation of complexity, this time regarding signals that are of multiple nature (audio-visual stimuli) ${ }^{13}$. Again, the fuzzy entropy function has been instrumental in reproducing human performance in the evaluation. From this examples and many others we easily see as the use of fuzzy measures, and more in general a fuzzy view of the evaluation problem concerning human performance can be seen as an adequate answer to the strongest point in Arnheim's critique of aesthetic evaluation: fuzziness lies on a different theoretical ground than the usual, sharp and statistical measurements, and results such as the ones briefly outlined above stand as a confirmation. The ample variety of technical instruments now available, such the different measures we have shown in the previous subsection, and of interpretative possibilities hinted in the previous section, corroborates the ideas presented in this and other papers: the concept of fuzziness, the tools already offered by Fuzzy Sets Theory and a straight-on implementation of the measures based on this concepts and ideas can be the first step for attaching and defeating Arnheim's doubts about the apparently illconceived krasis between order and structure. In some of our previous works (along the ones already mentioned see $9,29,12,39,38$ ) we have shown practical remedies to this doubts, algorithms to work out the kinks of computing the element of structural complexity, just one of the many that contribute in forming the idea of beauty. In this paper we have instead tried to debate a critical analysis that, maybe as a byproduct, can contribute discover and clarify 
new, not yet explored aspects of the relationship between the concept of information, in his most wide interpretation, and the ideas of vagueness and uncertainty.

\section{Conclusions}

As implicitly written in presenting our plan of work, this paper can be considered as a prolegomenon to a study of the possibilities offered by a fuzzy approach (or, perhaps, by a fuzzy context) for affording in a new way some classical and debated problems regarding science (mathematics) and art (aesthetic experience).

We hope that this paper can be useful in reconciling Birkhoff's clear-cut approach and Arnheim's structure criticism using FST as a bridge. Once fixed a reasonable context, general theoretical results are what one hopes to obtain. But the path for obtaining this can be very hard. Along the way, it can be fruitful to try experimenting the behavior of different formal tools strictly connecting the results with the analysis of the presuppositions implicit to the specific tools used. The necessity of experimenting in the development of fuzzy set theory, a common practice today ${ }^{7}$, has been recently heralded in 41,43 . Only at the end of a chain of new hypothesisverification and returning back it could emerge the profile of something very meaningful. And, in the shadow of it, the role that fuzzy ideas have played.

1. Rudolf Arnheim. Entropy and Art. University of California Press, Berkeley, CA, 1969.

2. J. D. Barrow. Art and science: Les liaisons dangereuses? In John Casti and Anders Karlqvist, editors, Art and Complexity. Elsevier B.V, 2003.

3. M. Bense. Aesthetica. Agis-Verlag, 1956.

4. D.E. Berlyne. Aesthetics and psychobiology. Century psychology series. Appleton-Century-Crofts, 1971.

5. G.D. Birkhoff. Aesthetic measures. Harvard University Press, 1933.

6. B. Bosanquet. A History of Aesthetic. Kessinger Publishing's rare reprints. Kessinger Publishing, 2005.

7. Maurizio Cardaci, Vito Di Gesù, Maria Petrou, and Marco Tabacchi. On the evaluation of images complexity: A fuzzy approach. In Isabelle Bloch, Alfredo Petrosino, and Andrea Tettamanzi, editors, Fuzzy Logic and Applications, volume 3849 of Lecture Notes in Computer Science, pages 305-311. Springer Berlin / Heidelberg, 2006.
8. Maurizio Cardaci, Vito Di Gesu, Maria Petrou, and Marco Elio Tabacchi. Attentional vs computational complexity measures in observing paintings. Spatial Vision, 22(3):195-209, 2009.

9. Maurizio Cardaci, Vito Di Gesu, Maria Petrou, and Marco Elio Tabacchi. A fuzzy approach to the evaluation of image complexity. Fuzzy Sets Syst., 160(10):1474-1484, 2009.

10. Maurizio Cardaci, Vito DiGesù, Maria Petrou, and Marco Elio Tabacchi. Attentional vs computational complexity measures in observing paintings. In Baingio Pinna, editor, Art and Perception - Towards a Visual Science of Art, part 2, Spatial Vision Perspectives, pages 319-333. VSP, an imprint of Brill, Leiden, Boston, 2008.

11. Maurizio Cardaci, Vito Di Gesù, Maria Petrou, and Marco Elio Tabacchi. Attentional vs computational complexity measures in observing paintings. In Baingio Pinna, editor, Art and Perception - Towards a Visual Science of Art, part 2, Spatial Vision Perspectives, pages 319-333. VSP, an imprint of Brill, Leiden, Boston, 2008.

12. Maurizio Cardaci and Marco Elio Tabacchi. Effects of internal texture on cognitive preferences concerning rotational symmetry. Sistemi Intelligenti, 3:441-454, 2009.

13. Floriana Carmeci, Marco Elio Tabacchi, Vito DiGesù, and Maurizio Cardaci. Effetti della complessità attenzionale di brani musicali sulla percezione temporale. In Atti del congresso della Sezione di Psicologia Sperimentale dell'Associazione Italiana di Psicologia, Cagliari, 2005. AIP.

14. R. Carnap. Logical Foundations of Probability. Chicago University Press, 1950.

15. A. De Luca and Settimo Termini. On the convergence of entropy measures of a fuzzy set. Kybernetes, 6(3):219-227, 1977.

16. Aldo De Luca and Settimo Termini. A definition of a non probabilistic entropy. Information and Control, 20(4):301-312, 1972.

17. Aldo De Luca and Settimo Termini. Entropy and energy measures of a fuzzy set. In Madan M. Gupta, Rammohan K. Ragade, and Ronald R. Yager, editors, Advances in Fuzzy Set Theory and Applications, pages 321-338. North-Holland, 1979.

18. Aldo De Luca and Settimo Termini. Entropy measures in fuzzy set theory. In Madhusudan G. Singh, editor, Systems and Control Encyclopedia, pages 1467-1473. Pergamon Press, 1988.

19. B R Ebanks. On measures of fuzziness and their representations. J. Math. Anal. and Appl., 94:24-37, 1983.

20. Hans J Eysenck and Maureen Castle. Training in art as a factor in the determination of preference judgements for polygons. British Journal of Psychology, 61(1):65-81, 1970. 
21. G.T. Fechner. Vorschule der Aesthetik. Number v. 1 in Vorschule der Aesthetik. Breitkopf \& Härtel, 1876.

22. B.R. Gaines. Foundations of fuzzy reasoning. International Journal of Man-Machine Studies, 8(6):623668, 1976.

23. C A Garabedian. Birkhoff on aesthetic measure. Bull. Amer. Math. Soc., 40(1):7-10, 1934.

24. Joseph Goguen. Fuzzy sets and the social nature of truth. Advances in Fuzzy Set Theory and Applications, pages 49-68, 1979.

25. G Greenfield. On the origins of the term "computational aesthetics". In L Neumann, M Sbert, B Gooch, and W Purgathofer, editors, Computational Aesthetics 2005: Eurographics Workshop on Computational Aesthetics, Graphics, Visualization and Imaging Girona, Spain, 2005.

26. Arthur O Lovejoy. The great chain of being: A study of the history of an idea. Transaction Publishers, 2011.

27. K. Menger. Ensembles flous et fonctions aléatoires. CR Acad. Sci. Paris, 322:2001-2003, 1951.

28. K. Menger. Probabilistic theories of relations. In Proceedings of the National Academy of Sciences of the United States of America, volume 37, page 178. National Academy of Sciences, 1951.

29. Maria Petrou, Marco Elio Tabacchi, and Roberta Piroddi. Networks of Concepts and Ideas. The Computer Journal, 53(10):1738-1751, 2010.

30. Baingio Pinna and Marco Tabacchi. A fuzzy approach to the role of symmetry in shape formation: The illusion of the scalene triangle. In Vito Di Gesù, Sankar Pal, and Alfredo Petrosino, editors, Fuzzy Logic and Applications, volume 5571 of Lecture Notes in Artificial Intelligence, pages 197-204. Springer BerlinHeidelberg, 2009.

31. J Rigau, M Feixas, and M Sbert. Conceptualizing Birkhoff's aesthetic measure using shannon entropy and Kolmogorov complexity. In Proc. Eurographics Workshop Computational Aesthetics, Graphics, Visualization and Imaging, pages 105-112. Eurographics Assoc., 2007.

32. J Rigau, M Feixas, and M Sbert. Informational aesthetics measures. IEEE Computer Graphics and Applications, pages 24-34, 2008.

33. R Scha and R Bod. Computational aesthetics. Informatie en Informatiebeleid, 11(1):54-63, 1993.

34. Rudolf Seising. The Fuzzification of Systems. The Genesis of Fuzzy Set Theory and Its Initial Applications - Developments up to the 1970s. Springer, 2007.

35. Rudolf Seising. On the absence of strict boundaries - vagueness, haziness, and fuzziness in philosophy, science, and medicine. Applied Soft Computing, 8(3): 1232 - 1242, 2008.

36. Theodor Skolem. A set theory based on a certain 3valued logic. Mathematica Scand., 8:127-136, 1960.

37. G Stiny and J Gips. Algorithmic aesthetics: computer- models for criticism and design in the arts. University of California Press, 1978.

38. Marco Elio Tabacchi. Fist: a physics-based operator for planar symmetry preferences. Cognitive Systems, 7(3):329-338, 2012.

39. Marco Elio Tabacchi and Settimo Termini. Measures of fuzziness and information: some challenges from reflections on aesthetic experience. In Proceedings of WConSC 2011, 2011.

40. Marco Elio Tabacchi and Settimo Termini. Varieties of vagueness, fuzziness and a few foundational (and ontological) questions. In Proceedings of EusFLAT 2011, Advances in Intelligent Systems Research, pages 578-583. Atlantis Press, 2011.

41. Marco Elio Tabacchi and Settimo Termini. A few remarks on the roots of fuzziness measures. In Salvatore Greco, Bernadette Bouchon-Meunier, Giulianella Coletti, Mario Fedrizzi, Benedetto Matarazzo, and RonaldR. Yager, editors, Advances in Computational Intelligence, volume 298 of Communications in Computer and Information Science, pages 62-67. Springer Berlin Heidelberg, 2012.

42. Settimo Termini. Do uncertainty and fuzziness present themselves (and behave) in the same way in hard and human sciences? In Eyke Hullermeier, Rudolf Kruse, and Frank Hoffmann, editors, IPMU 2010, volume 81 of CCIS, pages 334-343. Springer-Verlag, 2010.

43. Settimo Termini. Concepts, theories, and applications: the role of "experimentation" for formalizing new ideas along innovative avenues. In Enric Trillas, P Bonissone, L Magdalena, and J Kacprycz, editors, Combining Experimentation and Theory - a Hommage to Abe Mamdani, volume 271 of Studies in Fuzziness and Soft Computing, pages 359-370. Springer Berlin - Heidelberg, 2012.

44. Settimo Termini. On explicandum versus explicatum. In R. Seising and Veronica Sanz Gonzales, editors, Soft Computing in Humanities and Social Sciences, volume 273 of Studies in Fuzziness and Soft Computing, pages 113-124. Springer Berlin, 2012.

45. Settimo Termini. On some "family resemblances" of fuzzy set theory and human sciences. In Rudolf Seising and Veronica Sanz Gonzales, editors, Soft Computing in Humanities and Social Sciences, volume 273 of Studies in Fuzziness and Soft Computing, pages 3954. Springer Berlin, 2012.

46. Settimo Termini and Marco Elio Tabacchi. Fuzzy set theory as a methodological bridge between hard science and humanities. International Journal of Intelligent Systems, 29(1):104-117, 2014.

47. H Weyl. Symmetry. Princeton University Press, Princeton, New Jersey, 1989.

48. D J Wilson. An experimental investigation of birkhoff's aesthetic measure. Journal of Abnormal and Social Psychology, 34:390-394, 1939. 
49. S Wolfram. Universality and complexity in cellular automata. Physica D: Nonlinear Phenomena, 10(1):1-35, 1984.

50. Lotfi A. Zadeh. From circuit theory to system theory. Proceedings of the IRE, 50:856-865, 1962.

51. Lotfi A. Zadeh. Fuzzy sets. Information and Control,
8:338-353, 1965.

52. Bertrand Zavidovique, Vito DiGesu, and Marco Elio Tabacchi. Symmetry as a genuine dynamic visual feature. Symmetry, 2:554-581, 2010.

53. W H Zurek. Algorithmic randomness and physical entropy. Physical Review A, 40(8):4731-4751, 1989. 